\title{
DYNAMICS OF THE LEVEL OF TOTAL ELECTRON CONTENT DISTURBANCE AT HIGH AND MIDDLE LATITUDES ACCORDING TO GPS DATA
}

\author{
N.P. Perevalova , I.K. Edemsky, O.V. Timofeeva, D.D. Katashevtseva, A.S. Polyakova \\ Institute of Solar-Terrestrial Physics SB RAS, Irkutsk, Russia \\ pereval@iszf.irk.ru
}

We study the level of total electron content (TEC) disturbance in ionospheric mid-latitude and highlatitude regions, which occurred during 2013. TEC behavior is calculated using data from two GPS stations: MOND (Mondy) and NRIL (Norilsk). TEC variations are estimated from dual-frequency phase measurements for all radio signal paths. We analyze the TEC variations in two time ranges: 10 and 40 min. These ranges correspond to medium- and large-scale ionospheric disturbances respectively. The TEC disturbance level is characterized using a special index $W_{T E C}$. It allows us to receive multi-day continuous series of average TEC variation intensity. We reveal that at high latitudes $W_{\text {TEC }}$ variations correlate well with AE ones. The correlation between $W_{T E C}$ and $D_{s t}$ variations is much lower. The minimum level of TEC disturbance is independent of the season in the Arctic region; diurnal $W_{T E C}$ variations are more pronounced for medium-scale ionospheric disturbances than for large-scale ones. At mid-latitudes, the $W_{T E C}$ variation concurs with $D_{s t}$ and $K_{p}$ variations only during strong magnetic storms. The minimum level of TEC disturbance is higher in summer than in winter. At middle latitudes, the sunset terminator generates gravity waves. In the Arctic region, terminator-induced waves are not observed.

Keywords: GPS, ionosphere, total electron content, Arctic region, geomagnetic variations.

\section{INTRODUCTION}

One of the effective instruments for studying the ionosphere is represented by global navigation systems GPS and GLONASS with extensive networks of ground-based receivers. Ionospheric sounding by means of GPS/GLONASS radio signals enables us to examine ionospheric plasma irregularities. In recent years, much attention is being given to the study of morphology of ionospheric disturbances of various scales in separate regions of the globe [Tsugawa, Saito, 2004; Kotake et al., 2006; Tsugawa et al., 2007a, b; Otsuka et al., 2011, 2013]. General characteristics of traveling ionospheric disturbances (TID, 10-60-min periods) over Europe, Japan, and North America have been established. Daytime TIDs in these regions often occur in winter and during equinoxes. Considering the predominant direction (southward and southeastward) of daytime TIDs, the authors of [Kotake et al., 2006; Tsugawa et al., 2007a, b; Otsuka et al., 2011, 2013] suppose that these disturbances are generated by gravity waves; and averagely southwestward nighttime TIDs can be driven by electrodynamic forces. In Japan, general characteristics of TIDs with more than 60-min periods have been revealed [Tsugawa, Saito, 2004]. Three 
types have been separated: decaying and growing TIDs in a disturbed period and decaying TIDs in a quiet period. Average decay rates, periods, wavelengths, horizontal velocities, and propagation directions were determined for TIDs of each type.

The findings on the morphology of ionospheric disturbances were largely obtained from data acquired by dense GPS networks. However, such studies can also rely on data from individual GPS stations because even one station can monitor total electron content (TEC) variations in the ionosphere in a radius 500-1000 $\mathrm{km}$ due to spatial distribution in GPS receiver-satellite lines of sight (LOSs) within 24 hours. In this case, a serious limitation of the GPS ionospheric sounding technique is a short duration of continuous TEC series bounded by the observation time of one satellite (around 2-6 hr). This problem hampers examination of long-term TEC variations and their relationships with processes in Earth's magnetosphere, and solar wind effects. To solve this problem, ISTP SB RAS has devised a method allowing us to obtain multi-day continuous series of average intensity of TEC variations, filtered in a selected time range, from measurements made by one GPS station (the disturbance index $W_{\mathrm{TEC}}$ ) [Berngardt et al., 2014a, b; Voeykov et al., 2016]. In the present paper, this method is utilized to compare TEC variations in mid- and high-latitude regions with varying geomagnetic activity indices.

\section{MEASUREMENT DATA AND METHODS FOR THEIR PROCESSING}

In this study, we have used TEC variation series for 2013 obtained from data acquired at the midlatitude station MOND, included into the ISTP SB RAS-developed Siberian network of GPS/GLONASS receivers, as well as at the high-latitude GPS station NRIL that belong to the Geophysical Survey SB RAS (GS SB RAS) and is located at Norilsk Complex Magnetic-Ionospheric Station of ISTP SB RAS. Temporal resolution of the data is $1 \mathrm{~s}$.

Time series of TEC variations $I(t)$ were calculated from dual-frequency phase measurements in all GPS receiver-satellite LOSs for a chosen GPS station and for elevation angle of $>5^{\circ}$ to a satellite. The algorithm of TEC determination from phase measurements of a GPS receiver at two frequencies is well known and has been widely used over the past 20 years [Hofmann-Wellenhof et al., 1992; Afraimovich, Perevalova, 2006]:

$$
I=\frac{1}{40.308} \frac{f_{1}^{2} f_{2}^{2}}{f_{1}^{2}-f_{2}^{2}}\left[\left(L_{1} \lambda_{1}-L_{2} \lambda_{2}\right)+\text { const }+\sigma\right]
$$

where $L_{1} \lambda_{1}, L_{2} \lambda_{2}$ are increments of radio signal phase path caused by a phase delay in the ionosphere; $L_{1}$, $L_{2}$ are phase measurements of a GPS receiver at frequencies $f_{1}$ and $f_{2}$ respectively; const is ambiguity of phase measurements; $\sigma$ is a phase measurement error. Phase measurements enable us to compute TEC variations rather than an absolute value because the initial TEC value (const) remains unknown. The const value may be determined by diverse methods [Hofmann-Wellenhof et al., 1992]. When studying ionospheric disturbances from $I(t)$ series, a trend (data filtration) is eliminated; then const is simply ruled out. At the same time, phase measurements in a GPS system are carried out with a high degree of accuracy so that the error in determining TEC variations from dual-frequency phase measurements does not exceed 
$10^{14} \mathrm{~m}^{-2}$ (or 0.01 TECU) [Hofmann-Wellenhof et al., 1992; Afraimovich, Perevalova, 2006]. This permits detection and examination of fairly weak TEC disturbances arising from both natural and anthropogenic sources. A commonly-accepted unit of TEC measurement is TECU (Total Electron Content Unit). 1 TECU $=10^{16}$ electron $/ \mathrm{m}^{2}$.

The general level of TEC variation disturbances was estimated from the $W_{\text {TEC }}$ index. The method for calculating $W_{\text {TEC }}$ had several modifications [Ishin et al., 2009; Berngardt et al., 2014a, b; Voeykov et al., 2016]. In our work, we utilize the last version described in [Voeykov et al., 2016]. Below is a brief description of this method.

Initial data used for determining $W_{\mathrm{TEC}}$ are series of "oblique" TEC variations $I_{i}(t)$ along LOSs from a GPS receiver to an $i$ th GPS satellite, as well as series of $\theta_{i}(t)$ elevation angles of the $i$ th GPS satellite. The $I_{i}(t)$ series are filtered by a moving-average method in a given time range to provide filtered series $d I_{i}(t)$. The series of filtered "oblique" TEC variations $d I_{i}(t)$ are transformed into equivalent "vertical" values $d I_{\mathrm{v}, i}(t)$ [Voeykov et al., 2016]:

$$
d I_{\mathrm{v}, i}(t)=d I_{i}(t) \sin \left(\theta_{i}(t)\right)
$$

Next, for each series $d I_{\mathrm{v}, i}(t)$ the intensity $A_{i}(t)$ of TEC oscillations is found. For this purpose, the $d I_{\mathrm{v}, i}(t)$ variations are taken in modulus and smoothed by a moving average [Voeykov et al., 2016]:

$$
A_{i}(t)=\frac{1}{T} \int_{-T / 2}^{T / 2}\left|d I_{\mathrm{v}, i}(t+\tau)\right| d \tau,
$$

where $T$ is a smoothing window length that is usually chosen equal to the filtration period of initial TEC series.

Then, for each instant of time the TEC variation intensity $W_{\mathrm{TEC}}(t)$ averaged over all receiver-satellite LOSs for the selected GPS receiver is calculated. The method uses a procedure of weighted averaging of $A_{i}(t)$ series to avoid the sharp difference between average data in the time ranges corresponding to the beginning and end of observation at one satellite [Voeykov et al., 2016]:

$$
W_{\mathrm{TEC}}(t)=\frac{\sum_{i=1}^{N} A_{i}(t) S_{i}(t)}{\sum_{i=1}^{N} S_{i}(t)}
$$

where $N$ is the number of satellites, $S_{i}(t)$ is a weight function for the $i$ th satellite, which is calculated for each $A_{i}(t)$ series providing that the function $S_{i}(t)$ and its derivative must become zero at the end of the series. As a weight function we employ [Voeykov et al., 2016]

$$
S_{i}(t)=\sin ^{2}\left(\pi\left(t-t_{0, i}\right) / \Delta T_{i}\right)
$$

where $\Delta T_{i}$ is a length of relative intensity series $A_{i}(t) ; t_{0, i}$, its beginning. The $S_{i}(t)$ function is 1 in the middle of the series and monotonously decreases to 0 at the end of the series. 
This yields a long-term continuous series of average TEC variation intensities filtered in the chosen time range. The length of the $W_{\mathrm{TEC}}(t)$ series is arbitrary and can be from several hours to many days. The temporal resolution of the $W_{\mathrm{TEC}}(t)$ series is equal to the temporal resolution of a receiver. According to Formulas (3) and (4), $W_{\mathrm{TEC}}$ is an average modulus of TEC oscillation amplitude. Therefore, the dimension of $W_{\mathrm{TEC}}$ coincides with that of TEC; i.e. the measuring unit of $W_{\mathrm{TEC}}$ is TECU. Ranges of $W_{\mathrm{TEC}}$ variation are determined by ranges of TEC variations that very much depend on oscillation periods, i.e. on scales of ionospheric irregularities. As inferred from decades-long studies (see References in [Afraimovich, Perevalova, 2006]), for medium-scale TIDs (with 1-10-min periods) the modulus of TEC oscillation amplitude (and consequently of $W_{\mathrm{TEC}}$ ) can vary from 0 to 3 TECU; for large-scale TIDs (with 10-min periods) the amplitude modulus (and $W_{\mathrm{TEC}}$ ) ranges from 0 to $10 \mathrm{TECU}$.

In this paper, we deal with TEC oscillations in two time ranges: $1-10$ and 1-40 min that correspond to medium-scale and large-scale TIDs. The dynamics of TEC disturbance level is compared with that of the geomagnetic indices $A E, K_{\mathrm{p}}$, and $D_{\mathrm{st}}$ taken from the International Data Center in Kyoto [http://wdc.kugi.kyoto-u.ac.jp]. The indices represent intensity and behavior of the geomagnetic field. The low-latitude index $D_{\text {st }}$ contains information about planetary disturbances. It is calculated from magnetic field measurements made at four subequatorial stations; in the first approximation the $D_{\text {st }}$ variation can be considered as a ring current field [http://wdc. kugi.kyoto-u.ac.jp]. The high-latitude index $A E$ characterizes current intensity in the auroral zone and indicates substorm activity. It is computed from data obtained at observatories located in auroral and subauroral latitudes [http://wdc.kugi.kyoto-u.ac.jp]. $K_{\mathrm{p}}$ is a planetary index and is computed using data from mid-latitude geomagnetic observatories [http://www.swpc.noaa.gov].

\section{THE LEVEL OF TEC DISTURBANCE AT HIGH LATITUDES}

To comparatively analyze the level of TEC and geomagnetic field disturbances, we plotted time variations in $W_{\mathrm{TEC}}$ for 1-10 and 1-40-min disturbances and in magnetic indices $A E$, $D_{\text {st }}$, and $K_{\mathrm{p}}$ for each month of 2013 for the high-latitude station NRIL. Examples of variations in the $W_{\text {TEC }}, A E, D_{\text {st }}$, and $K_{\mathrm{p}}$ indices in March, June, September, and December 2013 are given in Figure 1.

Referring to Figure 1, $a$, a sudden storm commencement (SSC) was registered on March 15, 2013. In this case, $W_{\text {TEC }}$ values were small, $A E$ increased only to $\sim 250 \mathrm{nT}$. The storm did not reveal itself in $D_{\mathrm{st}}$ and $K_{\mathrm{p}}$ variations. During the strongest storm of this month on March 17, the $W_{\text {TEC }}$ index significantly rose up to $\sim 1$ and $\sim 1.5$ TECU for 10 and 40-min oscillations respectively. During the same day, the magnetic index $A E$ increased to $\sim 1750 \mathrm{nT}, D_{\text {st }}$ decreased to $-125 \mathrm{nT}$. This suggests a close correlation between variations in the magnetic indices and $W_{\mathrm{TEC}}$. In addition, we can single out disturbances of $A E$ on March $1-2,20-21,22-24$, and 27-30 followed by an increase in the $W_{\text {TEC }}$ index.

A strong magnetic storm was recorded on June 1, 2013 (Figure 1, b) with $K_{\mathrm{p}}=7, D_{\mathrm{st}} \approx-125 \mathrm{nT}$, $A E \approx 1250 \mathrm{nT}$ (SSC on May 31). During this period, $W_{\mathrm{TEC}}$ rose to $\sim 1.2$ and 2 TECU for 10 and 40 -min oscillations respectively. From June 6 to 8 there also occurred noticeable magnetic disturbances. 
March 2013
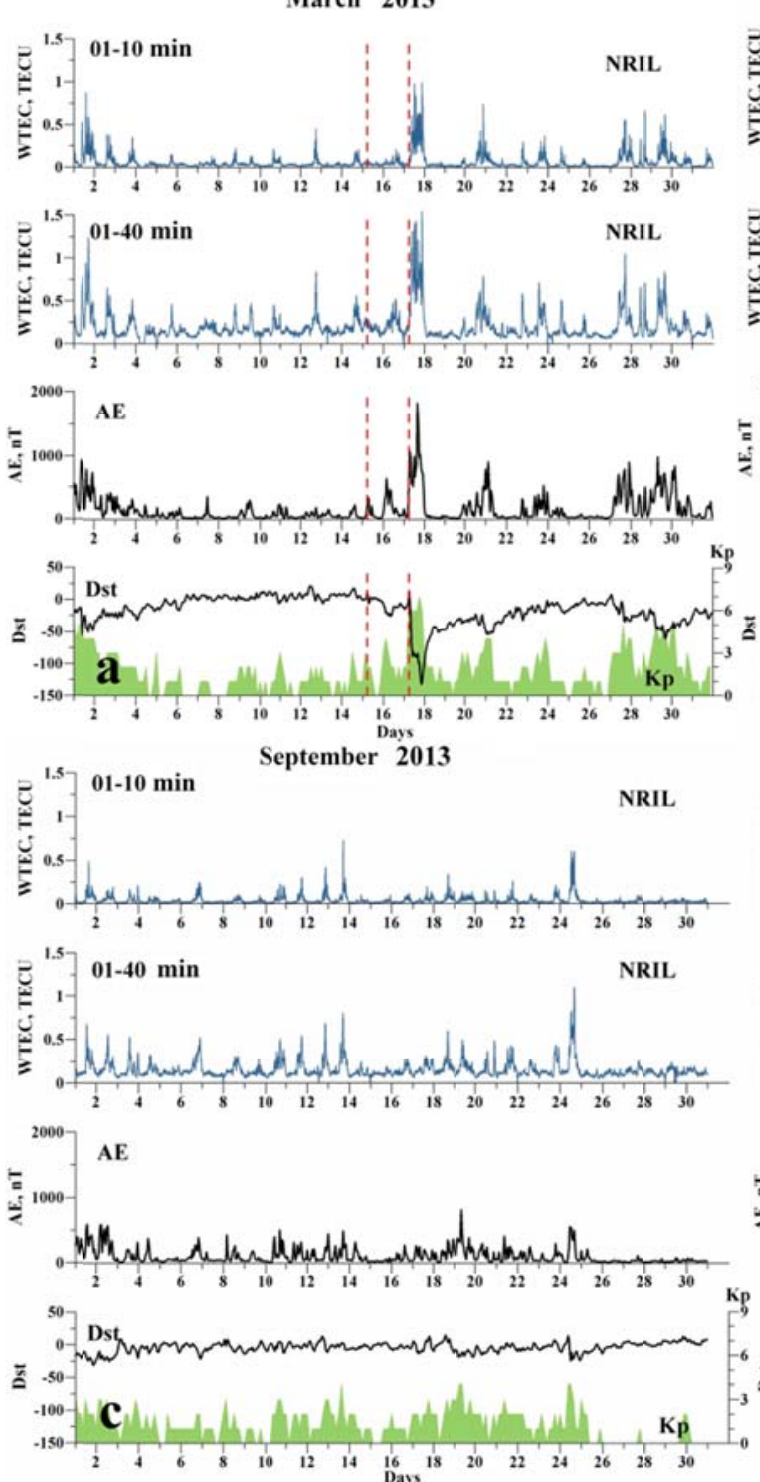

June 2013
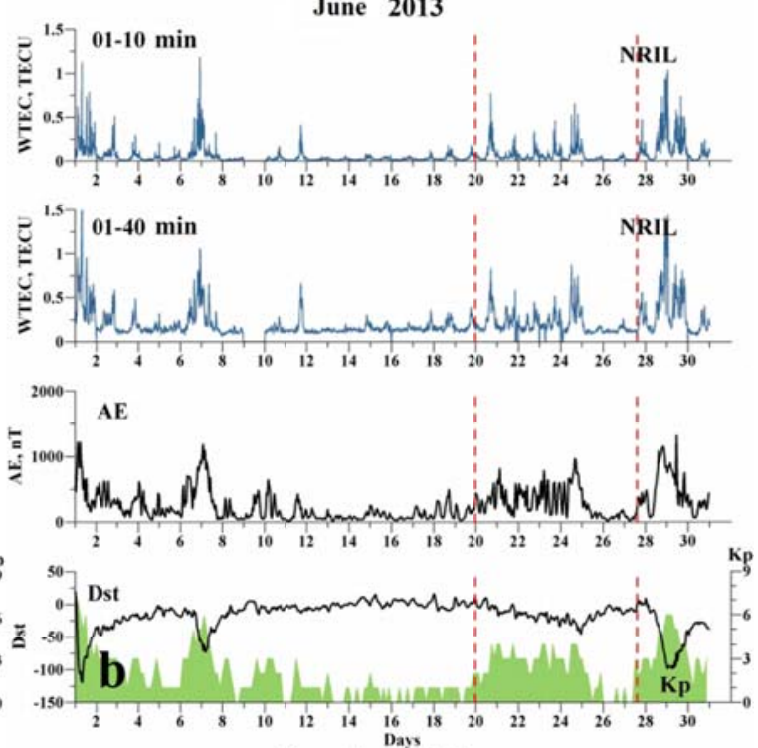

December 2013
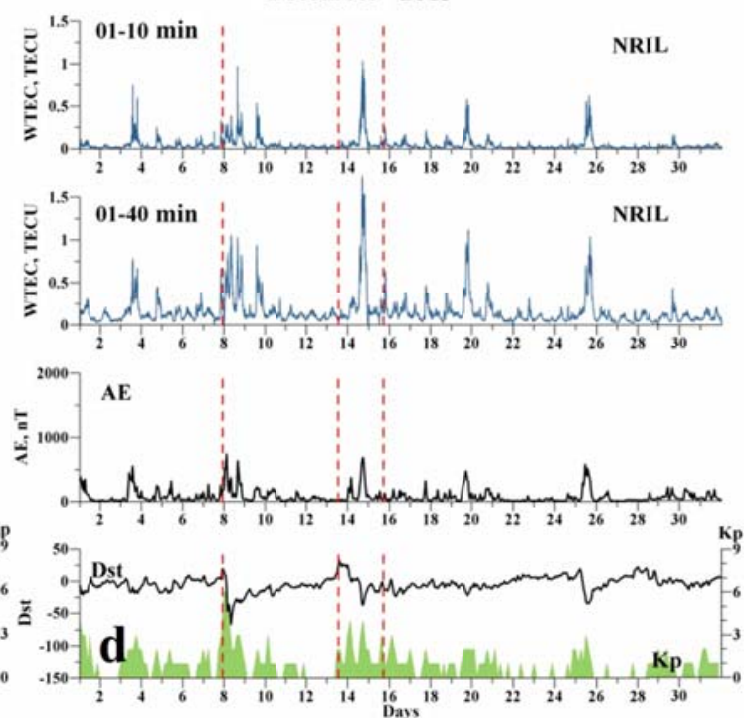

Figure 1. $W_{\text {TEC }}$ variations for 1-10 and 1-40-min disturbances for NRIL; $A E$, $D_{\text {st }}$, and $K_{\mathrm{p}}$ variations in March (a), June (b), September (c), and December $(d)$ 2013. Vertical dashed lines indicate sudden geomagnetic storm commencements

$A E$ rose to $\sim 1250 \mathrm{nT}, D_{\text {st }}$ was $-75 \mathrm{nT}$, and $K_{\mathrm{p}}=6$. Yet $W_{\text {TEC }}$ increased to $\sim 1.2$ TECU (10-min periods) and $\sim 1$ TECU (40-min periods). Between June 20 and 26 (SSC on June 19) significant oscillations of $A E$ ( $A E$ was as high as $\sim 1000 \mathrm{nT}$ ) were observed. During this period there was a close correlation between $W_{\text {TEC }}$ and $A E$ variations. In this case, the $D_{\text {st }}$ index varied insignificantly. A strong magnetic storm occurred on June 28-29 (SSC on June 27) with $D_{\text {st }} \sim-100 \mathrm{nT}$ and $K_{\mathrm{p}}=6$. It showed up in the enhancement of $A E$ to $\sim 1200 \mathrm{nT}, W_{\mathrm{TEC}}$ to $\sim 1$ and $\sim 1.5 \mathrm{TECU}$ for 10 and 40 -min disturbances respectively.

In September 2013 (Figure 1,c), the magnetosphere was undisturbed: $K_{\mathrm{p}}$ was under 4, $A E$ did not exceed $600 \mathrm{nT}$ on average. The $W_{\mathrm{TEC}}$ index was low, but the $W_{\mathrm{TEC}}$ variation amplitude periodically increased to $0.5-0.7$ TECU concurrently with slight disturbances of the $A E$ index. Also noticeable are pronounced diurnal variations in $W_{\text {TEC }}$ throughout the month. 
A concurrent rise in $A E$ and $W_{\text {TEC }}$ was observed on December 3, 2013 (Figure 1, $d$ ) with $A E$ equal to $\sim 500 \mathrm{nT}$ and $W_{\text {TEC }}$ being $\sim 0.75$ TECU. During weak magnetic storms on December 7-9, 13-14, and 25 manifesting themselves in $D_{\text {st }}, K_{\mathrm{p}}$, and $A E$, the $W_{\mathrm{TEC}}$ index increased approximately to $0.5-1$ TECU (for 10-min periods) and 1-1.75 TECU (for 40-min ones).

It follows from the above that in the Arctic region the $W_{\mathrm{TEC}}$ variations closely match those of the $A E$ index characterizing geomagnetic conditions at high latitudes. During planetary-scale geomagnetic storms there also exists a connection between $W_{\mathrm{TEC}}, D_{\mathrm{st}}$, and $K_{\mathrm{p}}$ variations. In the absence of strong storms, $W_{\mathrm{TEC}}$ variations poorly correlate with those of $D_{\mathrm{st}}, K_{\mathrm{p}}$ as these indices largely represent geomagnetic disturbances at equatorial and middle latitudes.

The level of minimum TEC disturbance intensity in the Arctic region is close to the TEC determination error and does not depend on the season: in 2013 it was 0.013 and 0.089 TECU for 10 and 40-min respectively.

Figure 2 illustrates intensities of diurnal $W_{\mathrm{TEC}}$ variations for the 10 and 40-min filtration periods and of the $A E$ geomagnetic index during 2013. The analysis shows that in the high-latitude ionosphere diurnal $W_{\mathrm{TEC}}$ variations reveal themselves more vividly in medium-scale TEC disturbances (Figures 1;2,a) than in largescale ones (Figures 1;2,b). Diurnal variations in the intensity of medium-scale disturbances are most pronounced in March-April and September-October. The $A E$ index varies regardless of the time of day (Figure 2,c). Maximum $A E$ values were registered in May and June 2013.

\section{THE LEVEL OF TEC DISTURBANCE AT MIDDLE LATITUDES}

Figure 3 depicts variations in $W_{\mathrm{TEC}}$ for 1-10 and 1-40-min disturbances for MOND as well as in $A E$, $D_{\text {st }}$, and $K_{\mathrm{p}}$ in March, June, September, and December 2013.

The comparison between Figures 1 and 3 indicates that the average level of $W_{\mathrm{TEC}}$ disturbance at middle latitudes is lower than that at high latitudes about three times for 10-min disturbances and about two times for 40-min ones. The average minimum level of TEC disturbance intensity at middle latitudes, as at high ones, is close to the TEC determination error. For large-scale disturbances, we can single out low seasonal variations in the level: in winter it is around 0.03 TECU; in summer, its value rises to 0.07 TECU. For medium-scale disturbances, the average minimum level of TEC disturbance intensity is close to 0.01 TECU.

At middle latitudes, $W_{\mathrm{TEC}}$ variations are less correlated with variations in magnetic indices (in particular with $A E$ ) than at high latitudes. As seen from Figure 3, $a, b$, the correlation between dynamics of large-scale disturbances $W_{\text {TEC }}$ and magnetic indices is observed largely during the strong magnetic storms of March 17, June 1, and June 28-29 with $D_{\text {st }}$ decreasing below $\sim 100 \mathrm{nT}$ and $K_{\mathrm{p}}$ being above 6 . The $W_{\mathrm{TEC}}$ values for 40 -min disturbances increased to $\sim 0.4, \sim 0.35$, and $\sim 0.25$ TECU respectively during those days, while the level of medium-scale disturbances varied slightly. In general, the intensity of the $W_{\mathrm{TEC}}$ response to a strong magnetic storm at middle latitudes is lower than that at high latitudes $\sim 15$ times 
for 10-min disturbances and $\sim 4$ times for 40 -min disturbances. Mid-latitude magnetic storms mostly generate large-scale disturbances.

In September 2013 (Figure 3,c) under quiet geomagnetic conditions, the $W_{\mathrm{TEC}}$ behavior was also quiet: $W_{\mathrm{TEC}}$ did not on average exceed $\sim 0.1$ and $\sim 0.2 \mathrm{TECU}$ for 10 and 40 -min oscillations respectively.
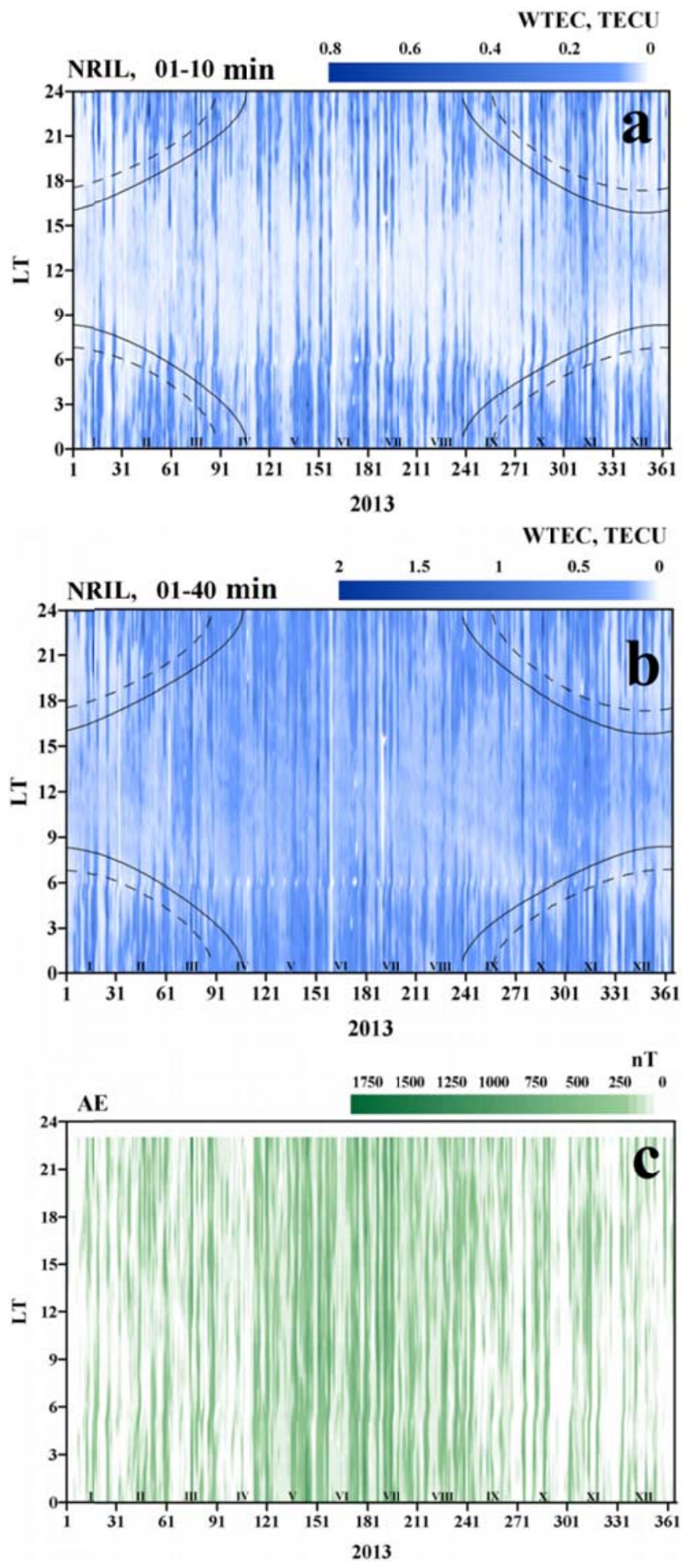

Figure 2. Intensities of diurnal variations in the disturbance index $W_{\mathrm{TEC}}$ for 1-10 (a) and 1-40-min (b) filtration periods as well as in the geomagnetic index $A E(c)$ during 2013 at high latitudes (NRIL). Lines indicate the time of solar terminator (ST) appearance at heights of $300 \mathrm{~km}$ (dashed line) and $100 \mathrm{~km}$ (solid line). Roman numerals denote months in 2013 

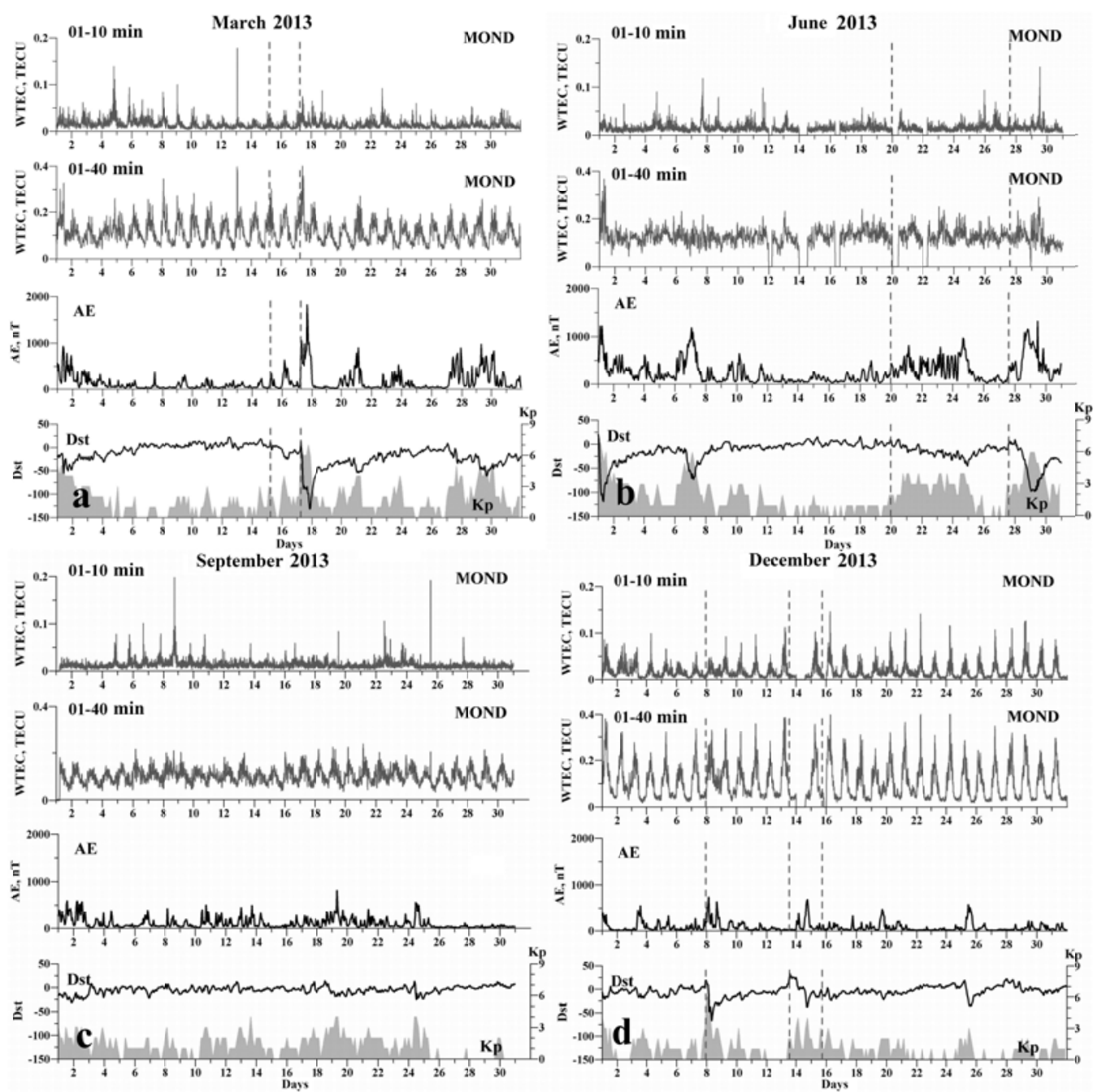

Figure 3. $W_{\mathrm{TEC}}$ variations for 1-10 and 1-40-min disturbances for MOND; $A E, D_{\mathrm{st}}$, and $K_{\mathrm{p}}$ variations in March $(a)$, June $(b)$, September $(c)$, and December $(d)$ 2013. Vertical dashed lines indicate sudden geomagnetic storm commencements

In December, the $W_{\mathrm{TEC}}$ variations exhibited high amplitude of diurnal variations marginally connected with geomagnetic variations. Even during December 8 and 16, 2013 storms $\left(D_{\mathrm{st}} \sim-50 \mathrm{nT}\right)$, the level of $W_{\text {TEC }}$ varied insignificantly.

Thus, at middle latitudes, the $W_{\mathrm{TEC}}$ response to geomagnetic variations is less pronounced than that at high latitudes. Variations in $W_{\mathrm{TEC}}, D_{\mathrm{st}}$ and $K_{\mathrm{p}}$ correlate during strong magnetic storms. In such periods, the intensity of large-scale TEC disturbance increases approximately two times in the daytime. Mediumscale TEC disturbances are slightly affected by magnetic field variations.

Figure 4 depicts intensities of diurnal $W_{\mathrm{TEC}}$ variations during 2013 for 10 and 40-min filtration periods at MOND. Diurnal $W_{\mathrm{TEC}}$ variations at middle latitudes have seasonal features (Figures 3, 4). In summer, disturbances are more intense at the beginning and end of the day; in other seasons, in the middle of the day. Notice an increase in variation intensity during daylight hours from October to the end of the year. 

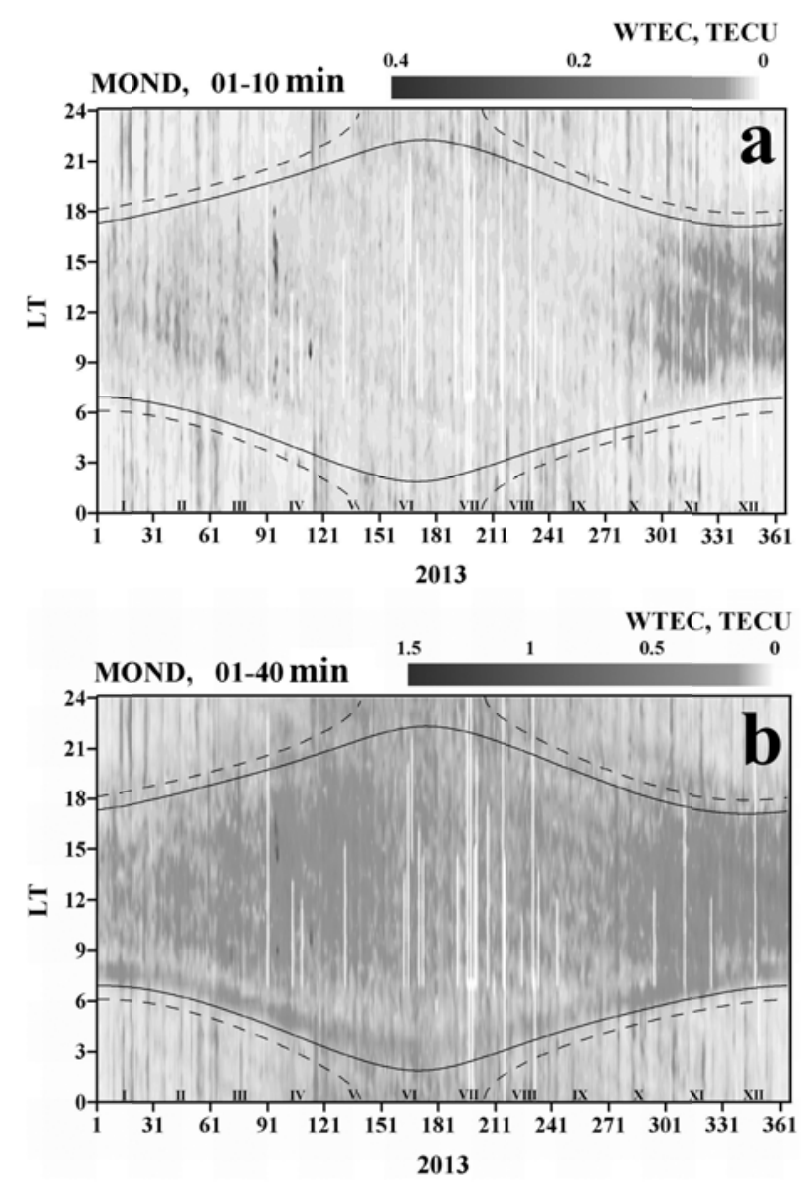

Figure 4. Intensities of diurnal $W_{\mathrm{TEC}}$ variations during 2013 for the 1-10 (a) and 1-40-min (b) filtration periods at middle latitudes (MOND). Lines indicate the time of ST appearance at heights of $300 \mathrm{~km}$ (dashed line) and $100 \mathrm{~km}$ (solid line). Roman numerals denote months in 2013.

Black lines in Figure 4 indicate the time of ST appearance at heights of 100 and $300 \mathrm{~km}$. Figure 4, $b$ shows that at middle latitudes (MOND) ST generates intense large-scale disturbances. ST-induced medium-scale disturbances are not observed (Figure 4, a). These results agree with the data [Afraimovich et al., 2009] establishing that at middle latitudes $\left(30-40^{\circ} \mathrm{N}\right)$ ST triggers disturbancesi with periods $T>15$ min, while disturbances with $T<10 \mathrm{~min}$ are not observed.

Taking this into account, we can conclude that ST generates gravity waves $(T>10$ min [Hocke, Schlegel, 1996]) and not acoustic ones ( $T<10 \mathrm{~min}$ [Hocke, Schlegel, 1996]). In the Arctic region (NRIL, Figure 2) there are no ST-driven wave disturbances of $W_{\text {TEC }}$.

\section{CONCLUSION}

We have used data from the mid-latitude GPS station MOND and high-latitude GPS station NRIL to analyze the general level of TEC disturbance (in time ranges 1-10 and 1-40 min) in 2013. It was characterized employing the special index $W_{\mathrm{TEC}}$ that provided multi-day continuous series of average intensity of TEC variations. The analysis we carried out has established the following features of 
variations in the TEC disturbance level.

In the Arctic region, the average minimum level of intensity of medium- and large-scale TEC disturbances is 0.013 and 0.089 TECU respectively. The minimum level of TEC disturbance intensity is seasonally independent. Diurnal $W_{\mathrm{TEC}}$ variations are more pronounced for medium-scale ionospheric disturbances ( $\sim 10$-min periods) than for large-scale ones ( $\sim 40$-min periods). $W_{\text {TEC }}$ variations are correlated well with $A E$ variations and less well with $D_{\mathrm{st}}$ and $K_{\mathrm{p}}$ variations; only during strong planetary-scale magnetic storms $W_{\mathrm{TEC}}$ variations concur with $D_{\mathrm{st}}$ ones. ST-generated wave disturbances of $W_{\mathrm{TEC}}$ are not observed.

At middle latitudes, the average minimum level of intensity of large-scale TEC disturbances varies from 0.03 TECU in winter to 0.07 TECU in summer. For medium-scale disturbances, it is close to 0.01 TECU. Diurnal $W_{\mathrm{TEC}}$ variations are pronounced throughout the year and have seasonal features. $W_{\mathrm{TEC}}$ variations at middle latitudes correlate well with $D_{\mathrm{st}}$ and $K_{\mathrm{p}}$ variations during strong magnetic storms. In this latitude range, ST causes large-scale disturbances (with periods over $15 \mathrm{~min}$ ), while medium-scale disturbances (with periods under $10 \mathrm{~min}$ ) are not observed. Thus, ST generates gravity waves and not acoustic ones.

We express profound gratitude to S.V. Voeykov and O.I. Berngardt for their method of calculating the $W_{\mathrm{TEC}}$ index, for the description of the method and the $W_{\mathrm{TEC}}$ calculation software, as well as for fruitful discussions. We also thank the Geophysical Survey SB RAS (GS SB RAS) for data from the GPS station NRIL. The study was supported by the RSF grant (Project No. 14-37-00027).

\section{REFERENCES}

Afrajmovich E.L., Perevalova N.P. GPS-Monitoring Verkhnej Atmosfery Zemli [GPS-Monitoring of the Earth's Upper Atmosphere]. Irkutsk, SC RRS SB RAMS. 2006, 480 p. (in Russian).

Afraimovich E.L., Edemsky I.K., Voeykov S.V., Yasuke-vich Yu.V., Zhivet'ev I.V. Spatio-temporal structure of the wave packets generated by the solar terminator. Adv. Space Res. 2009, vol. 44, no. 7, pp. 824-835.

Berngardt O.I., Voeykov S.V., Ratovsky K.G. Using a single GSP/GLONASS receiver for estimating the level of ionospheric disturbance. General Assembly and Scientific Symposium (URSI GASS), 2014 XXXI ${ }^{\text {th }}$ URSI, 16-23 Aug. 2014. IEEE. 2014a, pp.1-3. DOI: 10.1109/URSIGASS.2014. 6929809.

Berngardt O.I., Voeykov S.V., Ratovsky K.G. Using a single GPS/GLONASS receiver for estimating the level of ionospheric disturbance. The $40^{\text {th }}$ COSPAR Scientific Assembly. Moscow, Russia, August 2-10, 2014: abstracts. M., 2014b, P. C0.2-0015-14.

Hocke K., Schlegel K. A review of atmospheric gravity waves and travelling ionospheric disturbances $1982-$ 1995. Ann. Geophys. 1996, vol. 14, no. 5, pp. 917-940.

Hofmann-Wellenhof B., Lichtenegger H., Collins J. Global Positioning System: Theory and Practice. New York, Springer-Verlag Wien. 1992. 327 p.

Ishin A.B., Voeykov S.V., Perevalova N.P., Zalizovsky A.V., Sopin A.A. Ionospheric parameters' variations registered during powerful hurricanes near the USA Atlantic coast in 2005. Sovremennye problemy distantsionnogo zondirovaniya Zemli iz kosmosa [Present Problems of Remote Probing Earth From Space]. 2009. vol. 6, no. 2. pp. 274 279 (in Russian).

Kotake N., Otsuka Y., Tsugawa T., Ogawa T., Saito A. Climatological study of GPS total electron content variations caused by medium-scale traveling ionospheric disturbances. J. Geophys. Res. 2006, vol. 111, A04306. DOI: 10.1029/2005 JA011418. 
Otsuka Y., Kotake N., Shiokawa K., Ogawa T., Tsugawa T., Saito A. Statistical study of medium-scale traveling ionospheric disturbances observed with a GPS receiver network in Japan. Aeronomy of the Earth's Atmosphere and Ionosphere, IAGA Special Sopron Book Series 2. 2011. no. 21, pp. 291-299. DOI: 10.1007/978-94-007-0326-1_21.

Otsuka Y., Suzuki K., Nakagawa S., Nishioka M., Shiokawa K., Tsugawa T. GPS observations of mediumscale traveling ionospheric disturbances over Europe. Ann. Geophys. 2013, no. 31, pp. 163-172. DOI: 10.5194/angeo-31-163-2013.

Tsugawa T., Kotake N., Otsuka Y., Saito A. Medium-scale traveling ionospheric disturbances observed by GPS receiver network in Japan: a short review. GPS Solutions. 2007a, no. 11, pp. 139-144. DOI: 10.1007/s10291-006-0045-5.

Tsugawa T., Otsuka Y., Coster A. J., Saito A. Medium-scale traveling ionospheric disturbances detected with dense and wide TEC maps over North America. J. Geophys. Res. 2007b, vol. 34, L22101. DOI: 10.1029/2007GL031663.

Tsugawa T., Saito A. A statistical study of large-scale traveling ionospheric disturbances using the GPS network in Japan. J. Geophys. Res. 2004, vol. 109, A06302. DOI: 10.1029/2003JA010302.

Voeikov S.V., Berngardt O.I., Shestakov N.V. Using the index of disturbance of vertical TEC variations for studying ionospheric effects of the Chelyabinsk meteorite. Geomagnetizm i Ajeronomiya [Geomagnetism and Aeronomy]. 2016, no. 2 (Accepted) (in Russian).

URL: http://wdc.kugi.kyoto-u.ac.jp (accessed February 01, 2016).

The article is based on materials of the XIVth Young Scientists' Conference "Interaction of fields and radiation with matter" of Baikal Young Scientists' International School on Fundamental Physics (September 14-18, 2015, Irkutsk, Russia) 